Article

\title{
Biopolymer-Based Nanoparticles for Cystic Fibrosis Lung Gene Therapy Studies
}

\author{
Elena Fernández Fernández ${ }^{1, *}$, Beatriz Santos-Carballal ${ }^{2}$, Chiara de Santi $^{1}$ (D), \\ Joanne M. Ramsey ${ }^{3}$, Ronan MacLoughlin ${ }^{3,4,5}$ (D), Sally-Ann Cryan ${ }^{3}$ and Catherine M. Greene ${ }^{1}$ \\ (iD) \\ 1 Lung Biology Group, Department of Clinical Microbiology, Royal College of Surgeons in Ireland, \\ Beaumont Hospital, Dublin 9, Ireland; chiaradesanti@rcsi.ie (C.d.S.); cmgreene@rcsi.ie (C.M.G.) \\ 2 ChiPro GmbH, Anne-Conway-Straße 1, 28359 Bremen, Germany; bcarballal@chipro.de \\ 3 School of Pharmacy, Royal College of Surgeons in Ireland, Dublin 2, Ireland; joanneramsey@rcsi.ie (J.M.R.); \\ RMacLoughlin@aerogen.com (R.M.); scryan@rcsi.ie (S.-A.C.) \\ 4 School of Pharmacy and Pharmaceutical Sciences, Trinity College, Dublin 2, Ireland \\ 5 Aerogen Ltd., Galway Business Park, Dangan, Galway H91 HE94, Ireland \\ * Correspondence: elenaffernandez@rcsi.ie; Tel.: +353-01-809-3712
}

Received: 30 November 2017; Accepted: 8 January 2018; Published: 13 January 2018

\begin{abstract}
Lung gene therapy for cystic fibrosis disease has not been successful due to several challenges such as the absence of an appropriate vector. Therefore, optimal delivery of emerging therapeutics to airway epithelial cells demands suitable non-viral systems. In this work, we describe the formulation and the physicochemical investigation of biocompatible and biodegradable polymeric nanoparticles (NPs), including PLGA and chitosan (animal and non-animal), as novel methods for the safe and efficient delivery of CFTR-specific locked nucleic acids (LNAs).
\end{abstract}

Keywords: cystic fibrosis; cystic fibrosis transmembrane conductance regulator (CFTR); lung gene delivery; nanoparticles; biopolymers; PLGA; chitosan; Locked-Nucleic Acid (LNA)

\section{Introduction}

The most frequent lethal genetic disease in Caucasian populations is Cystic Fibrosis (CF). Mutations in the cystic fibrosis transmembrane conductance regulator (CFTR) gene cause abnormal ion transport in the epithelium of several tissues, which results in the production of abnormally thick and sticky mucus that blocks the organ, principally the lung, and is responsible for CF pathology. Chronic inflammation and recurrent bacterial infections are the result [1], leading to the progressive destruction of lung tissue and making pulmonary disease the primary cause of mortality in CF [2].

Correction of the defective CFTR gene is an attractive solution for this single-gene disease. Successful gene transfer formulations depend on two components which are: the therapeutic nucleic acid and a carrier molecule that binds to or contains that nucleic acid. However, clinical approaches for CF genetic therapies have mostly failed due to increased immune responses towards the vectors. Gene therapy focused on the use of viral carriers has been widely studied in CF treatments due to the high transfection efficiency reported [3]. Nevertheless, the use of viruses as vectors raises many concerns regarding possible immune responses, biosafety and severe inflammation after long periods of administration [4]. Therefore, non-viral vectors have emerged as a potentially safer alternative [5].

The use of biocompatible and biodegradable polymers such as chitosan or polylactide-co-glycolic acid (PLGA) is becoming more common for the next generation of nanoparticles. Cationic polymers bind to negatively charged nucleic acids through electrostatic interactions to form polyplexes. Chitosan is the main derivative of chitin, the second most abundant polysaccharide in nature. It is a linear biodegradable polysaccharide composed of randomly distributed $\beta(1-4)$-linked-D-glucosamine and 
$\mathrm{N}$-acetylglucosamine units. Chitosan exhibits several properties that make it an interesting material for pharmaceutical formulations. It induces low cytotoxicity, is biocompatible, biodegradable and mucoadhesive [6-8]. All these properties place chitosan as a potential alternative to administer therapeutics to the lung. The cationic properties of chitosan are useful in pharmaceutical formulations and biomaterials because the molecule can form polyelectrolyte complexes with negatively charged DNA, RNA, siRNA and microRNA [9]. It has been reported as a suitable candidate for transmucosal administration [10] and, after intratracheal administration to mice, chitosan complexes were found in the mid-airways and transgene expression was observed in epithelial cells [11]. Gene therapy based on the use of chitosan as a delivery vector has been extensively considered in the last decade [12], but few studies have assessed the use of chitosan as a potential gene delivery vector for CF [13-16].

Besides this natural polymer, the synthetic polymer PLGA is approved by the US Food and Drug Administration (FDA) and European Medicine Agency (EMA) in various drug delivery systems in humans [17]. PLGA polymers are widely used due to their inherent biocompatibility, low toxicity, immunogenicity, and biodegradability $[17,18]$. In vivo, PLGA is hydrolyzed to lactic acid and glycolic acid which are subsequently removed via the citric acid cycle. By altering the ratio of each monomer, different forms of PLGA can be produced with different rates of degradation. PLGA 50:50 (i.e., a copolymer whose composition is $50 \%$ lactide and $50 \%$ glycolide) is the most widely used copolymer, with the fastest biodegradation rate, which degrades in about 50 to 60 days [19]. PLGA drug carriers can achieve sustained cytoplasmic delivery via rapid escape from endolysosomes and can be used to generate a wide range of particles from nanoparticle $(\sim 10-1000 \mathrm{~nm})$ to microparticle $(1-250 \mu \mathrm{m})$ sizes. Consequently, PLGA nanoparticles encapsulating nucleic acids can be developed for both inhalation and targeting to the lower respiratory tract [20].

MicroRNAs (miRNA) are regulatory RNAs that negatively regulate target gene expression. CFTR is known to be regulated by a set of lead miRNAs which are overexpressed in the lungs of people with CF [21-24]. A new therapeutic strategy for CF involves cytoplasmic delivery of Locked Nucleic Acid (LNA)-modified RNA oligonucleotides custom-designed to block endogenous miRNAs binding to their cognate miRNA recognition elements (MREs) in the $3^{\prime}$ untranslated region (UTR) of the CFTR messenger RNA. These custom-designed inhibitors termed Target Site Blockers (also commonly referred to as morpholinos) do not have off-target effects associated with antimiR inhibitor approaches [25]. Anti-miRNA LNAs are antisense oligonucleotides that can be synthesized to be complementary to a mature miRNA of interest and inhibit its function [26]. Once introduced into cells, they act by masking the miRNA target site while not affecting the activity of the endogenous miRNA per se. LNAs possess an extremely high binding affinity to complementary RNA oligonucleotides, display improved mismatch discrimination and show high stability in biological systems [27].

Here we report a comprehensive investigation of polymeric nanoparticles (NPs) as a novel method for the safe and efficient delivery of CFTR-specific LNAs. To this aim, particles were prepared and characterized with state-of-the-art techniques, loading efficiency and stability in biological environment was assessed as well as their stability after nebulization.

\section{Results}

\subsection{Physicochemical Characterization by Dynamic Light Scattering (DLS) and Nanoparticle Tracking} Analysis (NTA)

NPs were prepared using PLGA loaded with specific LNA oligonucleotides at N/P ratio 4 following the protocol described by Kelly and coworkers [20]. Regarding NPs from chitosan sources, systems were prepared from a stock solution containing acetic acid and LNAs. Complexes with different $\mathrm{N} / \mathrm{P}$ ratios $(1.5,2,5,10,15)$ were formed.

A graphic representation of both types of systems is shown in Figure 1. Table 1 describes the physicochemical characteristics of the biopolymers. The LNAs used during this study (LNA1 and LNA2) were 16 bases in length with a $38 \%$ GC content. Since optimal particle size and colloidal stability 
in suspension are important aspects to consider, all NPs were fully characterized in terms of their average size diameter, polydispersity and surface charge (zeta potential).

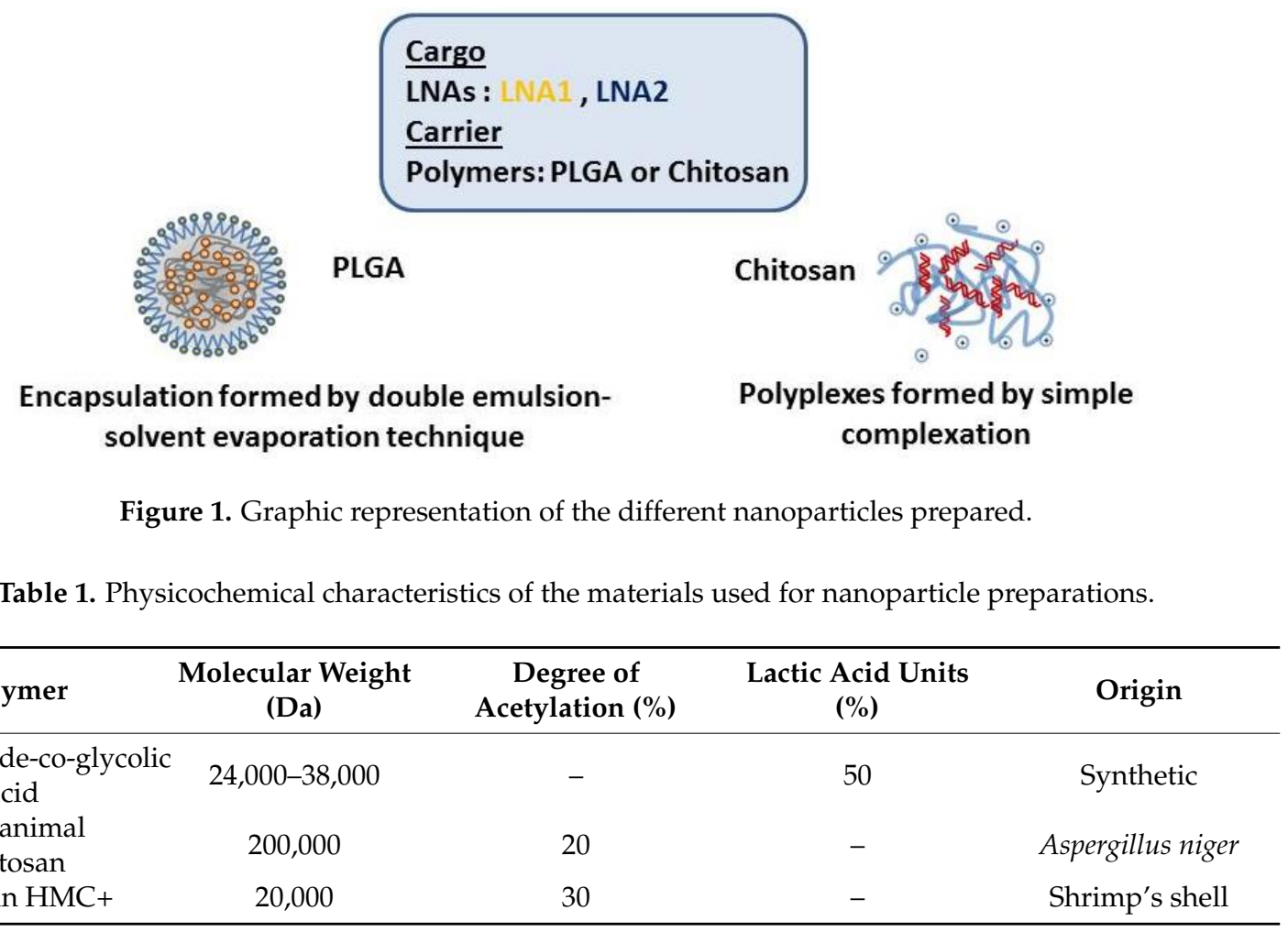

Figure 2 shows the values of average size diameter and polydispersity index (PDI) for the nanoparticles coated with PLGA obtained by DLS. The particle size oscillates between 180 and $300 \mathrm{~nm}$, and non-significant differences in size were found after the loading of the particles with the LNA. The PDI value was of approximately 0.1 for all the systems. It is suggested that PDI values below 0.3 are desired in order to ensure monodisperse nanoparticle formulations [28]. Higher size values were seen for particles encapsulating the LNA oligonucleotides. Specifically, PLGA particles loaded with LNA1 and with LNA2 present a Z-average diameter of $260 \pm 60 \mathrm{~nm}$ and $222 \pm 30 \mathrm{~nm}$, respectively. In comparison, Nanoparticle Tracking Analysis (NTA) measurements determined the size averages to be $166 \pm 2 \mathrm{~nm}$ and $168.3 \pm 0.3 \mathrm{~nm}$ respectively (Table 2 ). The measured $\zeta$-potential, corresponds to the difference in electric potential generated between the particle surface and the medium in which they are dispersed. Figure 3 depicts the variation of the zeta potential of PLGA NPs. Unloaded (blank) particles have a slightly negative $\zeta$-potential value. Particles loaded with LNA1 and LNA2 show a minor reduction in the $\zeta$-potential to values of around $-7 \mathrm{mV}$ for both.

Table 2. Comparison of the measurements performed by Dynamic Light Scattering (DLS) and Nanoparticle Tracking Analysis (NTA) regarding the average size diameter of the Locked-Nucleic Acid Nanoparticles (LNA-NPs).

\begin{tabular}{ccc}
\hline Nanoparticles & DLS & NTA \\
\hline PLGA + LNA1 & $260 \pm 60 \mathrm{~nm}$ & $166 \pm 2 \mathrm{~nm}$ \\
PLGA + LNA2 & $222 \pm 30 \mathrm{~nm}$ & $168.3 \pm 0.3 \mathrm{~nm}$ \\
Animal CS + LNA1 & $120 \pm 30 \mathrm{~nm}$ & $140 \pm 3 \mathrm{~nm}$ \\
Non-animal CS + LNA1 & $150 \pm 20 \mathrm{~nm}$ & $131.1 \pm 0.8 \mathrm{~nm}$ \\
Animal CS + LNA2 & $130 \pm 20 \mathrm{~nm}$ & $200 \pm 8 \mathrm{~nm}$ \\
Non-animal CS + LNA2 & $170 \pm 59 \mathrm{~nm}$ & $102 \pm 3 \mathrm{~nm}$ \\
\hline
\end{tabular}




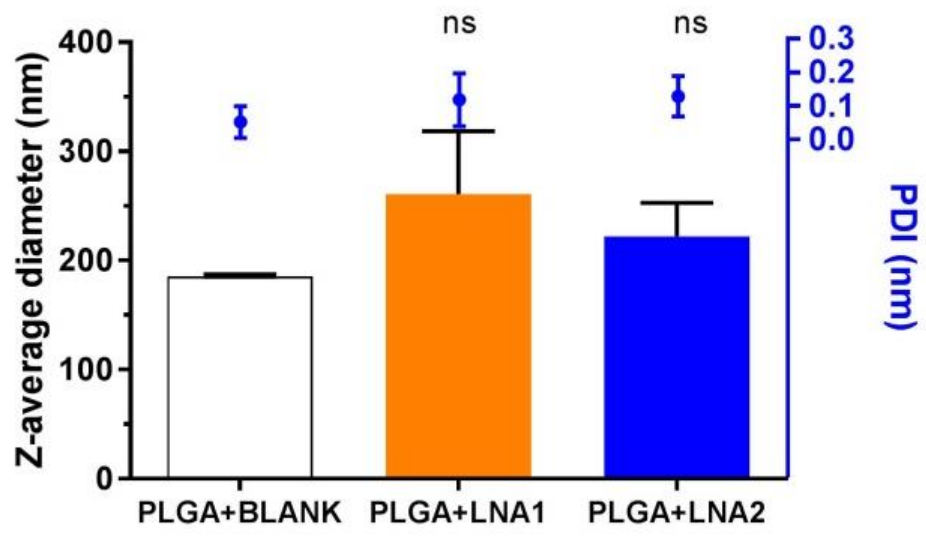

Figure 2. Variation of the Z-average size hydrodynamic diameter (nm) and polydispersity index (PDI) of PLGA NPs formed with LNA1 and LNA2. The values represented are the mean averages \pm SD of three independent experiments. Statistical comparisons were performed between unloaded and loaded PLGA particles using Kruskal-Wallis test for non-parametrical distribution $(p<0.05)$; "ns" stands for non-significant differences.

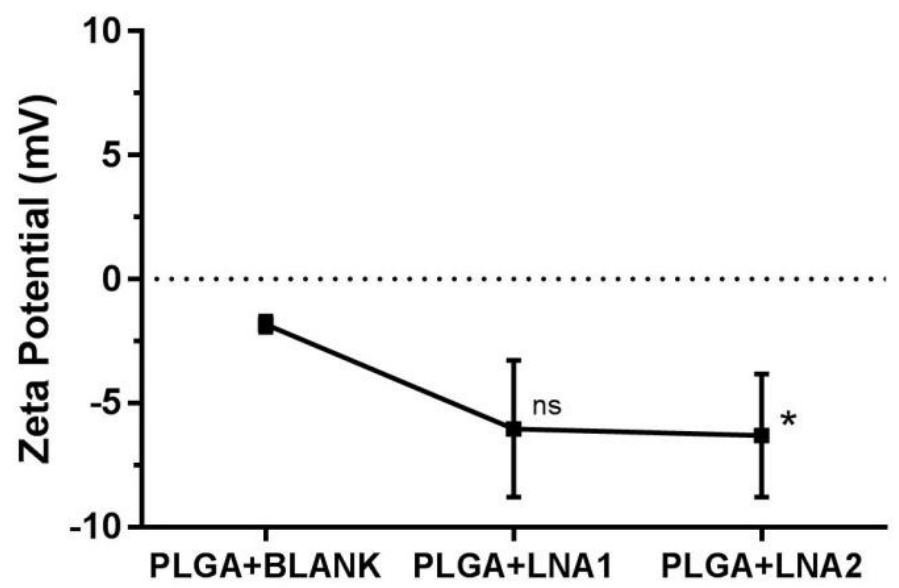

Figure 3. Variation of the zeta potential $(\mathrm{mV})$ of PLGA NPs formed with LNA1 and LNA2. The values represented are the mean \pm SD of three independent experiments. Statistical comparisons were performed between unloaded and loaded PLGA particles using Kruskal-Wallis test for non-parametrical distribution (* $p<0.05)$; "ns" stands for non-significant differences.

Likewise, the particles formed by simple coacervation processing using chitosans were characterized by DLS. Figure 4 shows two graphics corresponding to the average size hydrodynamic diameter and polydispersity index (PDI) of complexes formed with either LNA1 or LNA2. Complexes were designed under a range of positive $\mathrm{N} / \mathrm{P}$ ratio values, specifically, 1.5, 2, 5, 10, 15. DLS technique revealed that all the complexes formed with non-animal chitosan have a size below $200 \mathrm{~nm}$ and the small variations in size do not correlate to the different $\mathrm{N} / \mathrm{P}$ ratios. Complexes formed with chitosan from animal source loading LNA1 presented agglomerates at a N/P ratio of 1.5, and this is statistically significant. At ratio of 5 when bound to LNA2 the size increased up to $300 \mathrm{~nm}$. In the case of NTA measurements, all CS systems present an average size under $200 \mathrm{~nm}$ (see Table 2). The values of PDI in all cases are around 0.3, which indicates the formation of complexes with a monodisperse distribution of particle size for both CS types and LNAs. Figure 5 shows the observed $\zeta$-potentials in relation to $\mathrm{N} / \mathrm{P}$ ratio which varies from -20 to $+20 \mathrm{mV}$. All complexes with an N/P ratio of 10 showed a $\zeta$-potential of $+20 \mathrm{mV}$ and further addition of CS did not induce a significant increase in the zeta potential. 


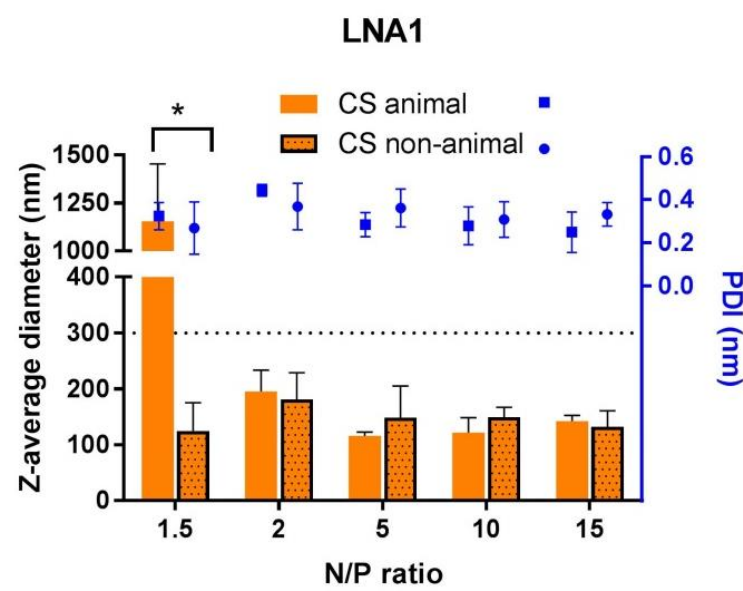

(A)

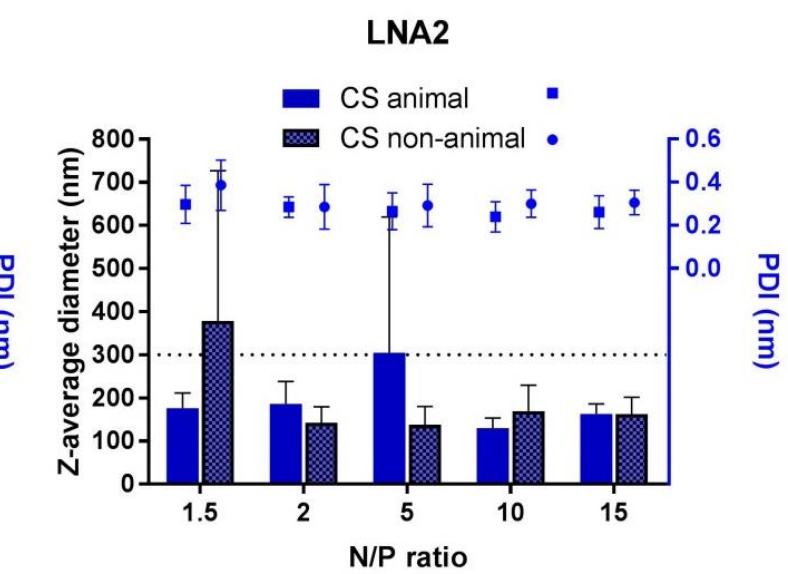

(B)

Figure 4. Variation of the Z-average size hydrodynamic diameter (nm) and polydispersity index (PDI) of chitosan (animal and non-animal) NPs formed with LNA1 (A) and LNA2 (B) at different N/P ratios $(1.5,2,5,10,15)$. The values represented are the mean averages $\pm S D$ of three independent experiments. Statistical comparisons were done between particles formed with either animal CS or non-animal CS within the same ratio and LNA. Statistical significance $\left.{ }^{*}\right)$ was determined using the Holm-Sidak method, with alpha $=5.0 \%$.

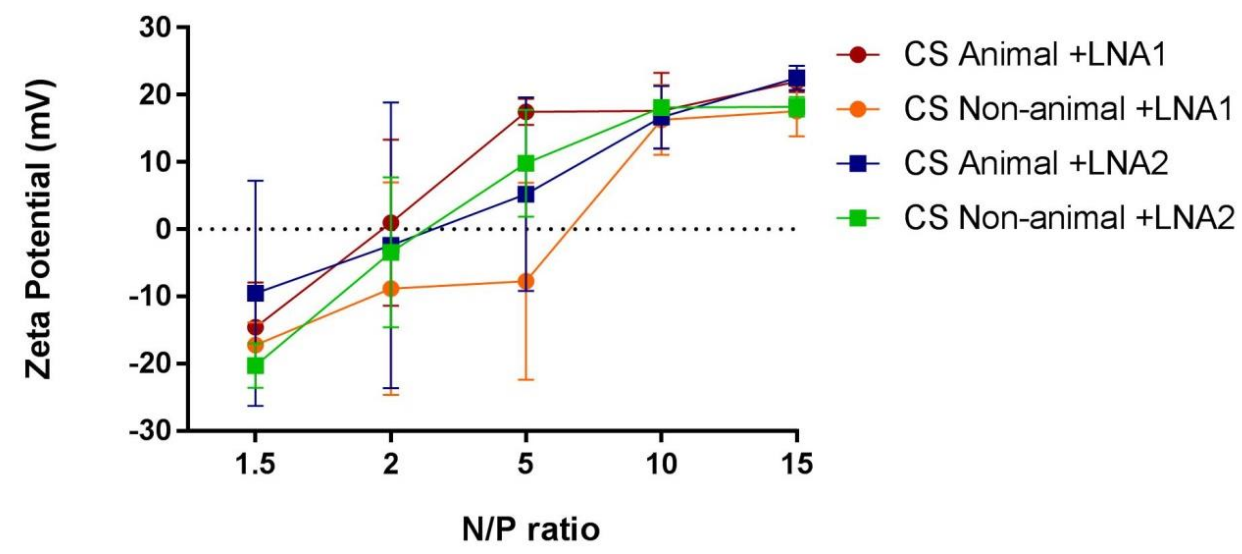

Figure 5. Variation of the zeta potential $(\mathrm{mV})$ of chitosan (animal and non-animal) NPs formed with LNA1 and LNA2 at varying N/P ratios $(1.5,2,5,10,15)$. The values represented are the mean \pm SD of three independent experiments

\subsection{Morphology of NPS}

Images of PLGA or CS NPs containing LNA1 and LNA2 were recorded by Transmission Electronic Microscopy (TEM) and analyzed in terms of particle morphology, size, and surface topology. Figure 6 shows representative images of PLGA containing LNA1 and LNA2. These values of size correlate well with those determined by Dynamic Light Scattering (DLS) and Nanoparticle Tracking Analysis (NTA). Figure 7 shows particles formed with all types of chitosans and LNAs at a ratio of 10 . All nanoparticles were spherical in shape showing its heterogeneous nature.

\subsection{Encapsulation Efficiency}

To accurately assess nucleic acid dosing and concentrations, the amount of each LNA encapsulated in the nanoparticles was determined by an RNA quantitation kit. This assay detects RNA in complex samples containing salts, free nucleotides, solvents, detergents, and protein. In our study, we have 
found that PLGA nanoparticle encapsulation efficiencies of LNA1 and LNA2 on average were found to be $70 \%$ and $20 \%$, respectively.

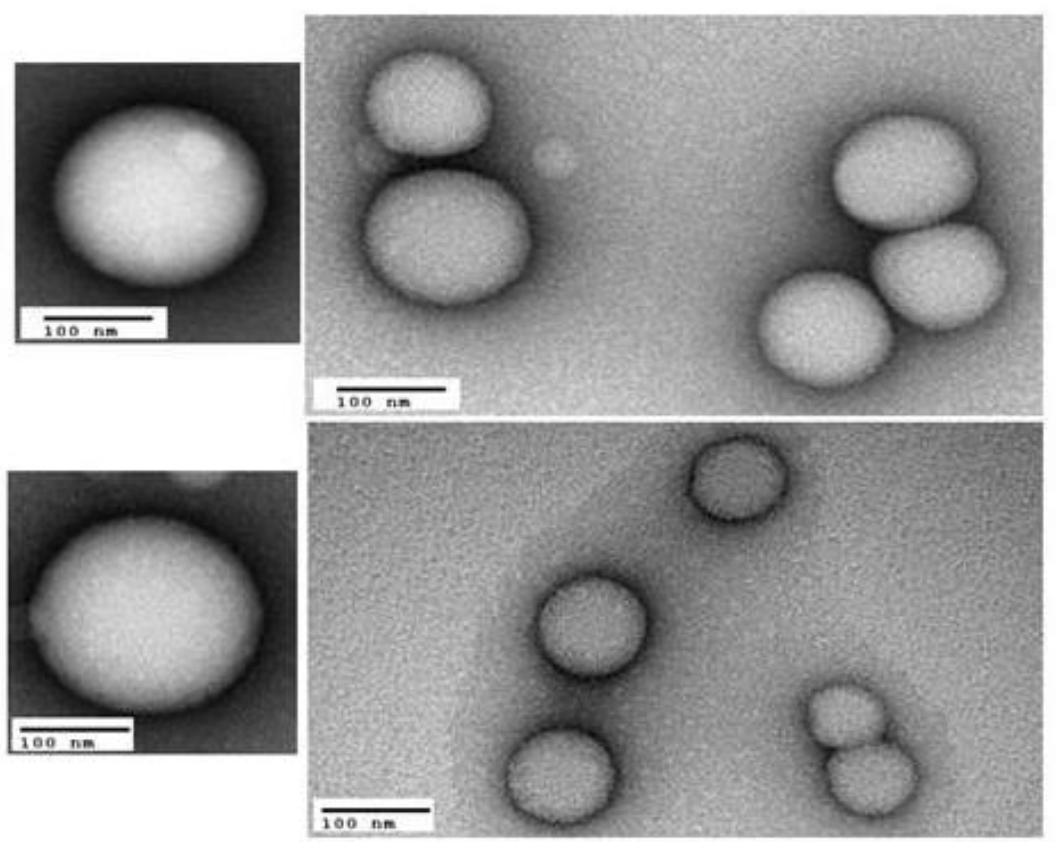

(A)

(B)

Figure 6. Representative TEM images of PLGA NPs containing (A) LNA1 and (B) LNA2 stained with UAR (Uranyl Acetate Replacement Stain).

\section{(A)}
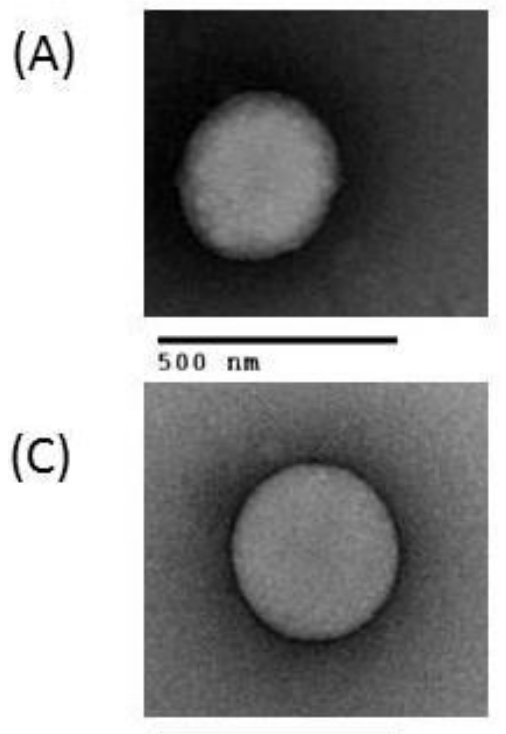

$500 \mathrm{~nm}$
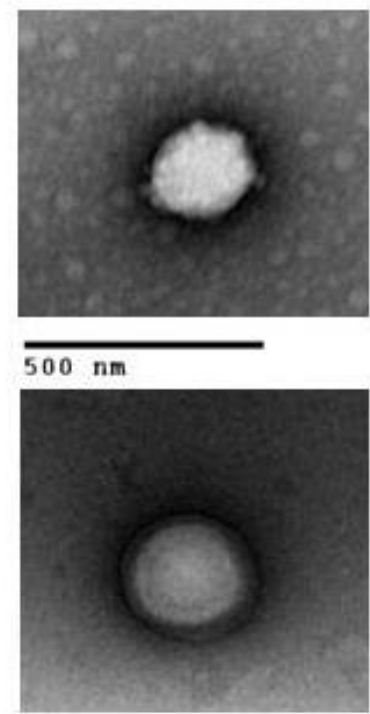

$500 \mathrm{~nm}$
(B)

(D)

Figure 7. Representative TEM images of CS NPs at N/P ratio of 10 containing: (A) LNA1 and (B) LNA2 formed with CS Animal; and (C) LNA1 and (D) LNA2 formed with CS non-animal. All CS NPS were stained with UAR (Uranyl Acetate Replacement Stain).

\subsection{Stability of CS-NPs in Biological Media}

The stability of different NP complexes during incubation in Opti-MEM containing HEPES and mannitol (equivalent to hypertonic conditions of $580 \mathrm{mM}$ ) was studied by DLS. Evolution of particle size distribution curves was analyzed after initial incubation at $37^{\circ} \mathrm{C}$ for $30 \mathrm{~min}$ and after every $15 \mathrm{~min}$ during $105 \mathrm{~min}$. Opti-MEM supplemented with HEPES and mannitol is recommended as a suitable 
transfection medium for Novafect (a CS-based commercial transfecting agent) from Novamatrix (Sandvika, Norway). At the same time, it is also well known that Opti-MEM is a commonly used medium to transfect epithelial cells [29]. Figure 8 shows the evolution of size over time for CS-LNA1 and CS-LNA2 from the two different CS sources. In general, an increase in average size diameter is observed when the particles are suspended in transfection medium. For particles formed with animal chitosan the diameter size increases over time from $\sim 300$ to $\sim 400 \mathrm{~nm}$. The opposite effect is observed for particles formed with non-animal chitosan, which have an initial particle size of $\sim 600 \mathrm{~nm}$ and over time they tend to stabilize and the size decreases to values of $\sim 450 \mathrm{~nm}$.

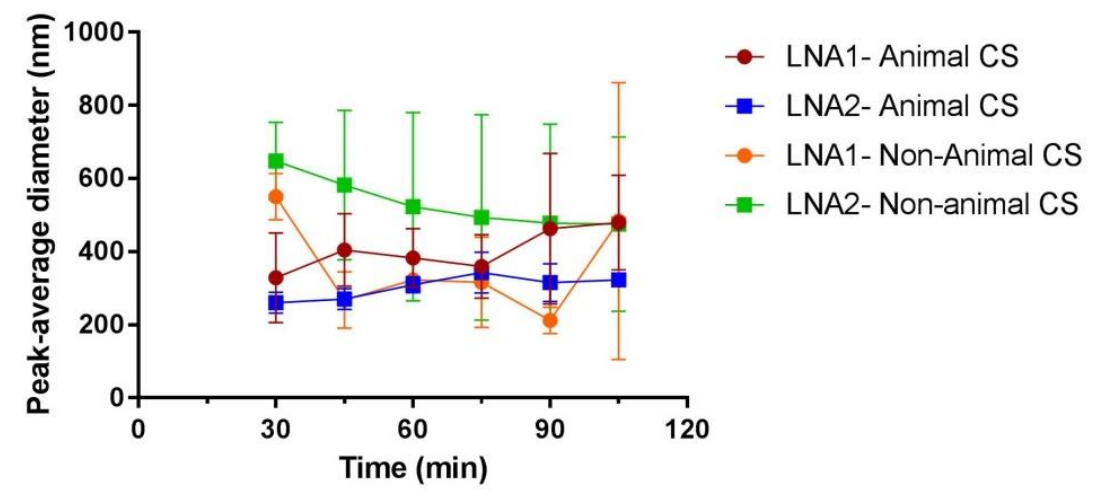

Opti-MEM+ Mannitol+HEPES

Figure 8. Stability of the LNA-CS NPs after initial incubation for $30 \mathrm{~min}$ and after every 15 min over the course of 105 min in Opti-MEM containing HEPES (4-(2-hydroxyethyl)-1-piperazineethanesulfonic acid) and mannitol at $37^{\circ} \mathrm{C}$. The values represented are the mean $\pm \mathrm{SD}$ of three independent experiments.

\subsection{NPs Nebulization}

To verify suitability for aerosolization the different biopolymers used in this study, PLGA, animal chitosan and non-animal chitosan NPs loaded with LNAs were aerosolized using the vibratingmesh type nebulizer, the Aerogen Solo (Aerogen, Galway, Ireland). No changes in size were detected in any case confirming that the nanoparticles were intact and not adversely affected by the process of nebulization using a vibrating mesh nebulizer. To test the quality of NPs post-nebulization, the particle size of all NPs pre- and post-nebulization were determined (Figure 9).

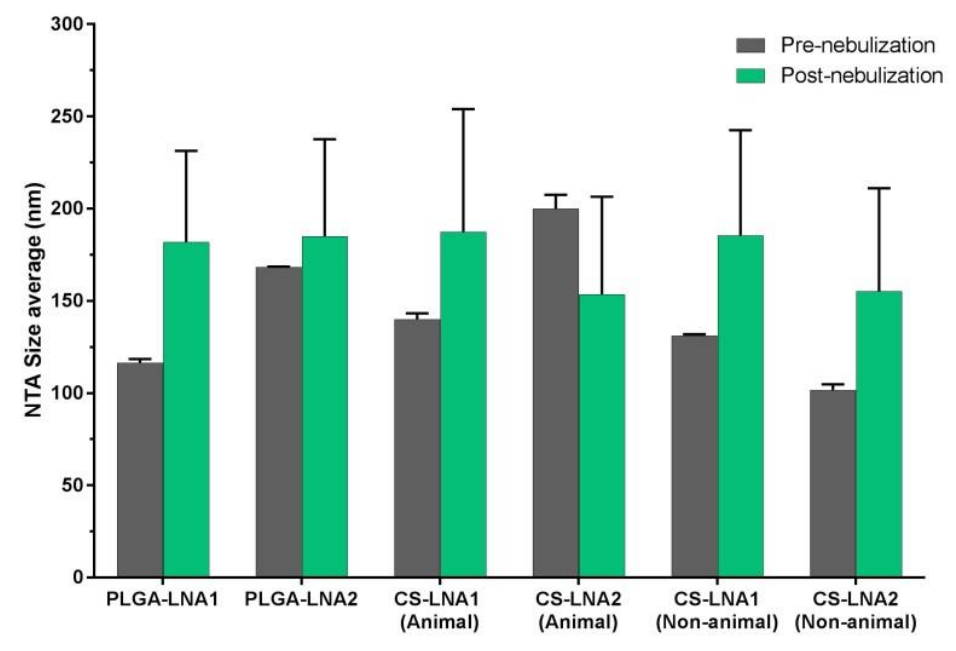

Figure 9. Effect of nebulization in LNAs-NPs. All LNA-NPs were measured before and after nebulization using NTA technique. The Aerogen Solo vibrating mesh nebulizer (Aerogen, Galway, Ireland) was used to generate the aerosol. 


\section{Discussion}

Successful gene delivery is among the main challenges in lung CF gene therapy. This is attributed to the negative charge and hydrophilic character of nucleic acids. These characteristics prevent them from entering cells by passive diffusion. Classically, gene therapy vectors are broadly classified as viral or non-viral, but the latest ones are less immunogenic and easier to manufacture [30]. To find a safe and efficient non-viral vector for CFTR-specific Locked Nucleic Acids (LNAs) delivery, in this study, we described here the use of biocompatible and biodegradable biopolymer-based nanoparticles (NPs). We demonstrate the successful formulation and physicochemical characterization of LNAs nanoparticles based on either synthetic or natural cationic polymers, such as PLGA and chitosan (CS) from animal or non-animal source. The potential for aerosol-mediated delivery was also assessed.

Nanoparticle size and surface properties are key parameters influencing a delivery system in terms of safety, release kinetics, as well as tissue penetration and cellular internalization [31,32]. It is well reported that efficient intracellular internalization via endocytosis requires positively charged particles of less than $500 \mathrm{~nm}$ [33]. Larger particles are reported to be phagocytosed by macrophages [34]. Our study shows that LNAs formed particles are in the range of $200 \mathrm{~nm}$ as measured by using the common and widespread method of Dynamic Light Scattering (DLS) but also using a cutting edge technique such as Nanoparticle Tracking Analysis (NTA). A comparison of the data acquired using the two techniques is shown in Table 2. LNA-PLGA NPs showed a slightly smaller size $(<200 \mathrm{~nm})$ when NTA is used in comparison with DLS $(\sim 200 \mathrm{~nm})$. In the case of LNAs-CS NPs, the average size observed for these systems is lower than $200 \mathrm{~nm}$ in all cases. It is well reported that NTA presents several advantages and benefits over DLS including the analysis of polydisperse systems and the concentration of nanoparticles. In addition, DLS measures changes in scattering intensity from a bulk sample, while NTA measures observed particle diffusion directly from particle-by-particle analysis [35]. NTA also has a substantially better peak resolution than DLS when the two methods are compared [36]. Likewise, images captured by TEM are in agreement with the results obtained by DLS and NTA. These images have allowed us to visualize the complexes and evaluate their morphology and spherical character.

Complexes formed with PLGA are all negatively charged, due to the presence of phosphate groups from the nucleic acids. Nonetheless, anionic systems have also been reported to produce successful therapeutic delivery [37]. Comparable evidence has already been reported in similar nanoparticles formed between PLGA and microRNA, where complexes presented a charge of $-21.4 \mathrm{mV}$ [38]. For particles formed with chitosans it is possible to observe that most of the complexes are negatively charged at low $\mathrm{N} / \mathrm{P}$ ratios. Values of zeta potential increase, almost in a monotonic manner, with increasing $\mathrm{N} / \mathrm{P}$ ratios. It is worth mentioning here that negative zeta potential values were not expected since this was previously calculated in order to produce positive complexes. However, it might be possible to argue that, because zeta potential is only an indicator of stability against agglomeration for charged stabilized systems, from our data we can speculate that complexes formed at low N/P ratios (e.g., below 5) might present a different conformation but they are still stable [39]. The trend observed in zeta potential values, might explain the increase in size for particles formed between LNA2 and animal chitosan at $\mathrm{N} / \mathrm{P}$ ratio of 5 . Under these conditions, zeta potential values reveal that the complexes are close to neutrality, the effect of repulsive forces is almost negligible and nanoparticles could have got together.

The formulation for PLGA NPs resulted in a high loading capacity for LNAs achieving levels of $70 \%$ and $20 \%$ for LNA1 and LNA2, respectively. Protocol optimization regarding the formulation of these PLGA NPs using the cationic lipid DOTAP based on previous studies carried out in our laboratory [20] allowed us to achieve these aforementioned levels of encapsulation efficiency. Such values are comparable to those reported in the literature using PLGA and siRNA [40].

Stability in transfection medium is a fundamental parameter for judging the behavior and effectiveness of particles in cell environment and later on their successful translation into clinical trials. In fact, there is a lack of systematic stability studies or methodologies for assessing the 
evaluation of this important parameter in in vitro experiments. The colloidal stability of NP in the cell environment will determine their biodistribution, pharmacokinetics, and systemic toxicity [41]. Here, it can be observed that chitosan nanoparticles remain relatively stable throughout the duration of the study e.g., for $105 \mathrm{~min}$. After this time, it is expected that particles would be attached to the cell membrane by electrostatic forces and afterwards starts the internalization process via endocytosis. The observed increase in particle size in transfection medium is related to interactions with the proteins and electrolytes present in such environment. The stabilization over at least 105 min is due to the presence of repulsive hydration forces, these have been previously reported by the authors for chitosan particles [15]. However, changes among our different samples can be due to many factors like the thickness of the absorbed protein layer formed onto the particles and the ionic strength of the medium. A more detailed study would be required in order to recognize the potential influence of these parameters.

Aerosolized therapeutic treatments represent the ideal route of administration for a variety of lung diseases such as asthma, chronic obstructive pulmonary disease, respiratory infection and lung cancer [42]. In fact, nanoparticle suspensions can be delivered to the lungs via inhalation using nebulizers [43]. However, recent data shows that nanoparticles can be also delivered as dry powder formulations [44-46]. In the specific context of cystic fibrosis gene therapy studies, it has been previously documented that NPs can deliver genes to the airway epithelium by nebulization $[47,48]$. Here LNA-NPs formulated in water and nebulized by an Aerogen Solo vibrating mesh type nebulizer, which facilitates high efficiency dose delivery of aerosol medications to the lung $[49,50]$, retained their biophysical properties in terms of particle size suggesting that aerosolized LNA-NPs may be suitable for cellular uptake and potentially useful for clinical application.

Regarding the polymers used, nanoparticles formulated from PLGA offer a low toxicity and it is reported as an efficient carrier system for the delivery of nucleic acids in cells [51]. Based on previous literature, PLGA particles enter the cell efficiently through specific and non-specific endocytosis mechanism and PLGA nanoparticles can slowly release the encapsulated cargo leading to therapeutic effect [52]. In addition, PLGA particles have previously been tested for the delivery to the lungs, including pharmaceuticals currently used for the treatment of inflammatory lung diseases such as $\mathrm{CF}$ and COPD [53].

In the case of the natural polymer, chitosan is particularly interesting as a gene carrier due to its biodegradability, biocompatibility, low toxicity and low immunogenicity [6]. It is well documented that chitosan is a biotechnologically suitable transfecting system for gene therapy purposes [54]. Our previous studies provided a proof-of-principle that chitosan from animal sources was a natural non-toxic vector able to co-transfect a well-established model cell line for CF [15]. To the best of our knowledge this is the first time the use of chitosan from a non-animal source as a potential therapeutic vector has been reported. Here, LNA-NPs from a non-animal chitosan source, specifically from Aspergillus niger cell wall constituents, were fully characterized at the physicochemical level demonstrating it to be a potential new resource and highlighting it as an alternative to chitosan derived from shellfish products.

\section{Materials and Methods}

\subsection{Preparation of CFTR-Specific LNAs-Loaded PLGA/DOTAP Nanoparticles}

LNA oligonucleotides (Exiqon) were encapsulated in DOTAP/PLGA nanoparticles using the double emulsion solvent evaporation (DESE) method as previously described [40,55]. To improve encapsulation efficiency LNA was condensed with a cationic lipid DOTAP at an N/P (defined as the molar ratio of amine to phosphate groups) ratio of 4:1 using a hydration of freeze-dried matrix (HFDM). Briefly, CFTR-specific LNAs were diluted in $200 \mu \mathrm{L}$ of RNA-free water and DOTAP was dissolved in $200 \mu \mathrm{L}$ of Tertbutanol. The LNA solution was added dropwise to the lipid mixture, mixed, and lyophilized overnight. PLGA 503H (Boehringer Ingelheim, Ingelheim am 
Rhein, Germany) was dissolved in dichloromethane (DCM) (2.9\% $w / v)$ and vortexed. Lyophilized LNA/DOTAP was resuspended in RNase-free water and PLGA solution and sonicated for a total of 3 bursts of $5 \mathrm{~s}$ in continuous pulses mode at 70\% amplitude to form the primary (water-in-oil $(w / o)$ ) emulsion. The primary emulsion was added dropwise to a $2 \%(w / v)$ poly(vinyl alcohol) (PVA) solution homogenizing on ice for $10 \mathrm{~min}$ in continuous pulses mode at $70 \%$ amplitude to form a secondary water-in-oil-in-water $(w / o / w)$ emulsion and then added to $2 \%(w / v)$ PVA. The emulsion was mechanically stirred in the fume hood over night to allow the solvent to evaporate and allow nanoparticle formation. The nanoparticles were collected by centrifugation at 30,000 $g$ for $25 \mathrm{~min}$ at $4{ }^{\circ} \mathrm{C}$. To remove residual PVA, nanoparticles were washed in distilled water and centrifuged thrice. Following this, samples were resuspended in RNase-free water. Nanoparticles (1 mg) were freeze-dried for $24 \mathrm{~h}$ in $1 \mathrm{~mL}$ RNAse-free water.

\subsection{Preparation of CFTR-Specific LNAs-Chitosan Nanoparticles}

Ultra-pure biomedical grade chitosans were used to prepare the nanoparticles. Non-animal chitosan from Aspergillus niger was used for the formulation and it was provided by ChiPro $\mathrm{GmbH}$ (Bremen, Germany) (Batch No. 0151222) with a DA $=20 \%$, Mw $=200 \mathrm{kDa}$ based on the manufacturer's specifications. Likewise, chitosan from an animal origin was provided by HMC+ (Halle, Germany; Code 70/5 Product No. 24200, Batch No. 212-170614-01; DA = 30\%, Mw = $20 \mathrm{kDa}$ based on the manufacturer's specifications).

The chitosans were dissolved in $1 \%$ acetic acid solution overnight at room temperature to a stock concentration of $2 \mathrm{mg} / \mathrm{mL}$, and then diluted with ultra-pure water to reach the desired concentration. A series of complexes were prepared at different charge (N/P) ratios, (defined as the molar ratio of amine to phosphate groups) by mixing the chitosan working solutions with a constant amount of LNA (Table 1). The mixtures were incubated for $30 \mathrm{~min}$ at room temperature to form the self-assembled complexes.

\subsection{Nanoparticle Characterization}

The size distribution of the NPs was determined by dynamic light scattering with non-invasive back scattering (DLS-NIBS) at an angle of 173 with an automatic attenuator setting. The zeta potential (Z) was determined from the electrophoretic mobility by mixed-laser Doppler electrophoresis and phase analysis light scattering (M3-PALS), using the well-known Henry's equation and Smoluchowski's approximation as reported in previous studies $[9,15,56]$. Samples were prepared as previously described before being diluted to $1 \mathrm{ml}$. Both parameters were measured using a Malvern Zetasizer ZS 3000 (Malvern, UK).

Nanoparticle tracking analysis (NTA) measurements were performed with a Nanosight NS300 (Malvern, UK). All suspensions were diluted in ultrapure water for measurement following equilibration. Samples were loaded into a laser module sample chamber which allowed for temperature control. Real time video analysis of the nanoparticles was recorded via an in-built sCMOS camera with computer controlled motorized focus. Automatic data analysis was performed on recorded data using the NTA 2.3 software.

\subsection{Encapsulation Efficiency of LNAs-PLGA NPS}

Nanoparticle yield was measured by weighing a 1:10 aliquot lyophilised in a pre-weighed, non-static microtube on an MX5 microbalance (Mettler Toledo, Columbus, OH, USA). For measuring encapsulation efficiency (EC) and loading capacity (LC), a 1:10 aliquot (approx. $1 \mathrm{mg}$ ) of lyophilised NPs was dissolved in $200 \mu \mathrm{L}$ chloroform for $10 \mathrm{~min}$ and subsequently vortexed at room temperature for $90 \mathrm{~min}$ after the addition of $500 \mu \mathrm{L}$ dissociation buffer (1X Tris-EDTA, $2 \mathrm{mg} / \mathrm{mL}$ heparin and $0.292 \mathrm{mg} / \mathrm{mL}$ octyl ß-D-glucopyranoside). The vortexed emulsion was centrifuged at $26,000 \times g$ for $15 \mathrm{~min}$ at $4{ }^{\circ} \mathrm{C}$. The aqueous layer was transferred to a new microtube and nucleic acid content was measured with the Quan-IT microRNA Assay kit (Thermo Fisher Scientific, Waltham, MA, USA). 
The measurements were controlled by performing parallel measurements with stock nucleic acid concentration dissolved in dissociation buffer.

$$
\begin{gathered}
\% \mathrm{EE}=\frac{\text { retrievednucleicacid }}{\text { loadednucleicacid }} \times 100 \\
\mathrm{LC}\left(\mu \mathrm{g}_{\text {nucleicacid }} / \mathrm{mg}_{\mathrm{NPs}}\right)=\frac{\text { retrievednucleicacid }(\mu \mathrm{g})}{\mathrm{NPs}(\mathrm{mg})}
\end{gathered}
$$

\subsection{NPs Morphology}

Nanoparticles were visualized by transmission electron microscopy (TEM) in order to further confirm size and determined the morphology. Briefly, LNAs-NPs were prepared as previously described. A drop of NPs suspension was placed on a silicon monoxide carbon-coated copper grid (200 mesh, Mason technologies, Deer Park, NY, USA). Samples were allowed to air dry for approximately 10-15 min before being negative stained with $2 \%$ uranyl acetate alternative (URA) solution. Excess stain was removed using filter paper and the grids allowed to air dry fully before analysis. Imaging was performed using a Hitachi H-7650 Transmission Electron Microscope (Hitachi High Technologies, Berkshire, UK) at $120 \mathrm{kV}$.

\subsection{Stability in Biological Media of LNAs-CS NPs}

The short-term stability of LNA-CS NPs was assessed by preparation of particles as above mentioned with $100 \mathrm{~mL}$ of Opti-MEM (Life Technologies, Hong Kong, China), supplemented with Mannitol and HEPES, and subsequently incubating them for $105 \mathrm{~min}$ at $37{ }^{\circ} \mathrm{C}$ under agitation. The stability was evaluated by measuring the evolution of the hydrodynamic size by DLS.

\subsection{Nebulization}

The LNAs-NPs were reconstituted in RNAse-free water at room temperature. Nebulization was conducted with the Aerogen Solo vibrating mesh nebulizer (Aerogen, Galway, Ireland). The NPs dispersions were nebulized into a $15 \mathrm{~mL}$ centrifuge tube affixed to the nebulizer and collected after nebulization was complete. The average particle size before and after nebulization was measured as described above using the NTA system.

\subsection{Statistical Analysis}

Results are expressed as means $\pm \mathrm{SD}$. Statistical analysis was carried out using GraphPad Software Prism v7 (San Diego, CA, USA). Experiments were statistically analyzed using non-parametric tests using the Kruskal-Wallis test and Holm Sidak test. Differences were considered statistically significant when $p$-values $\left(^{*}\right)<0.05$. All experiments were conducted at least in triplicate and with at least three technical replicates per experiment.

\section{Conclusions}

Here we reported a comprehensive investigation of polymeric nanoparticles (NPs) as a novel method for the delivery of CFTR-specific LNAs. To this end, we described the physicochemical characterization of LNA-NPs using different biocompatible and biodegradable polymers; synthetic poly lactide-co-glycolide (PLGA) and natural chitosan (CS). PLGA-LNA NPs were produced at $(+/-)$ charge ratio of 4 , while electrostatic self-assembled CS-LNA NPs from two sources (animal and non-animal) were complexed in a range of $(+/-)$ charge ratios (from 1.5 to 15). Size distribution, polydispersity index and zeta potential were determined by dynamic light scattering (DLS) and Nanoparticle Tracking Analysis (NTA). The average size diameter for PLGA-LNA NPs was $\sim 200 \mathrm{~nm}$, while CS-LNA NPs were $<200 \mathrm{~nm}$ regardless of CS source. Transmission electronic microscopy images confirmed previous findings. All NPs had a monodisperse particle size distribution. The surface charge 
was determined from the electrophoretic mobility which revealed a neutral to slightly negative surface charge in PLGA-LNA NPs. At higher $(+/-)$ charge ratios $(10,15)$, LNA-CS NPs reached a stable value of approximately $+20 \mathrm{mV}$. Encapsulation efficiency analysis revealed a high amount of LNA encapsulated by PLGA NPs. The LNA-CS NPs size slightly increased in stability studies performed using biological media. All LNA-NPs were nebulized, and no differences were found in terms of particle size with measurements prior nebulization. In conclusion, our work shows that CFTR-specific LNA biopolymer-based nanoparticles represent a promising system for further development of new lung-targeted CF therapeutic approaches.

Acknowledgments: Funding for this work is gratefully acknowledged from Cystic Fibrosis Foundation Therapeutics (USA). We thank ChiPro GmbH (Bremen, Germany) for providing the vegetal chitosan. The authors would like to thank Rachel Gaul for her technical support during nebulization studies and Brenton Cavanagh (RCSI, Dublin, Ireland) for his assistance in obtaining TEM images.

Author Contributions: E.F.F., S.-A.C. and C.M.G. conceived and designed the experiments. E.F.F. performed the experiments. E.F.F. and B.S.-C. analyzed the data. B.S.-C., C.d.S., J.M.R. and R.M. contributed reagents/materials/analysis tools. B.S.-C. helped to draft the paper. E.F.F. and C.M.G. wrote the paper.

Conflicts of Interest: The authors declare no conflict of interest.

\section{References}

1. Davis, P.B. Cystic fibrosis since 1938. Am. J. Respir. Crit. Care Med. 2006, 173, 475-482. [CrossRef] [PubMed]

2. Gibson, R.L.; Burns, J.L.; Ramsey, B.W. Pathophysiology and management of pulmonary infections in cystic fibrosis. Am. J. Respir. Crit. Care Med. 2003, 168, 918-951. [CrossRef] [PubMed]

3. Conese, M.; Ascenzioni, F.; Boyd, A.C.; Coutelle, C.; De Fino, I.; De Smedt, S.; Rejman, J.; Rosenecker, J.; Schindelhauer, D.; Scholte, B.J. Gene and cell therapy for cystic fibrosis: From bench to bedside. J. Cyst. Fibros. 2011, 10, S114-S128. [CrossRef]

4. Griesenbach, U.; Alton, E.W.F.W.W. Progress in gene and cell therapy for cystic fibrosis lung disease. Curr. Pharm. Des. 2012, 18, 642-662. [CrossRef] [PubMed]

5. Armstrong, D.K.; Cunningham, S.; Davies, J.C.; Alton, E.W.F.W. Gene therapy in cystic fibrosis. Arch. Dis. Child. 2014, 99, 465-468. [CrossRef] [PubMed]

6. Rinaudo, M. Chitin and chitosan: Properties and applications. Prog. Polym. Sci. 2006, 31, 603-632. [CrossRef]

7. Younes, I.; Rinaudo, M. Chitin and chitosan preparation from marine sources. Structure, properties and applications. Mar. Drugs 2015, 13, 1133-1174. [CrossRef] [PubMed]

8. Menchicchi, B.; Fuenzalida, J.P.; Bobbili, K.B.; Hensel, A.; Swamy, M.J.; Goycoolea, F.M. Structure of chitosan determines its interactions with mucin. Biomacromolecules 2014, 15, 3550-3558. [CrossRef] [PubMed]

9. Santos-Carballal, B.; Aaldering, L.J.; Ritzefeld, M.; Pereira, S.; Sewald, N.; Moerschbacher, B.; Götte, M.; Goycoolea, F.M. Physicochemical and biological characterization of chitosan-microRNA nanocomplexes for gene delivery to MCF-7 breast cancer cells. Sci. Rep. 2015, 5, 13567. [CrossRef] [PubMed]

10. Grenha, A.; Al-Qadi, S.; Seijo, B.; Remuñán-López, C. The potential of chitosan for pulmonary drug delivery. J. Drug Deliv. Sci. Technol. 2010, 20, 33-43. [CrossRef]

11. Koping-Hoggard, M.; Tubulekas, I.; Guan, H.; Edwards, K.; Nilsson, M.; Varum, K.M.; Artursson, P. Chitosan as a nonviral gene delivery system. Structure-property relationships and characteristics compared with polyethylenimine in vitro and after lung administration in vivo. Gene Ther. 2001, 8, 1108-1121. [CrossRef] [PubMed]

12. Gomes, C.P.; Ferreira Lopes, C.D.; Duarte Moreno, P.M.; Varela-Moreira, A.; Alonso, M.J.; Pêgo, A.P. Translating chitosan to clinical delivery of nucleic acid-based drugs. MRS Bull. 2014, 39, 60-70. [CrossRef]

13. Nydert, P.; Dragomir, A.; Hjelte, L. Chitosan as a carrier for non-viral gene transfer in a cystic-fibrosis cell line. Biotechnol. Appl. Biochem. 2008, 51, 153-157. [CrossRef] [PubMed]

14. McKiernan, P.J.; Cunningham, O.; Greene, C.M.; Cryan, S.-A.A. Targeting miRNA-based medicines to cystic fibrosis airway epithelial cells using nanotechnology. Int. J. Nanomed. 2013, 8, 3907-3915. [CrossRef]

15. Fernández, E.; Santos-Carballal, B.; Weber, W.-M.; Goycoolea, F.M. Chitosan as a non-viral co-transfection system in a cystic fibrosis cell line. Int. J. Pharm. 2016. [CrossRef] [PubMed]

16. Kolonko, A.K.; Fernández Fernández, E.; Santos-Carballal, B.; Goycoolea, F.M.; Weber, W.-M. Functional Restoring of Defect CFTR by Transfection of CFTR-mRNA Using Chitosan. JSM Genet. Genom. 2016, 3, 2. 
17. Danhier, F.; Ansorena, E.; Silva, J.M.; Coco, R.; Le Breton, A.; Préat, V. PLGA-based nanoparticles: An overview of biomedical applications. J. Control Release 2012, 161, 505-522. [CrossRef] [PubMed]

18. Kumari, A.; Yadav, S.K.; Yadav, S.C. Biodegradable polymeric nanoparticles based drug delivery systems. Colloids Surf. B Biointerfaces 2010, 75, 1-18. [CrossRef] [PubMed]

19. Makadia, H.K.; Siegel, S.J. Poly Lactic-co-Glycolic Acid (PLGA) as Biodegradable Controlled Drug Delivery Carrier. Polymers 2011, 3, 1377-1397. [CrossRef] [PubMed]

20. Kelly, C.; Yadav, A.B.; Lawlor, C.; Nolan, K.; O’Dwyer, J.; Greene, C.M.; McElvaney, N.G.; Sivadas, N.; Ramsey, J.M.; Cryan, S.A. Therapeutic Aerosol Bioengineering of siRNA for the Treatment of Inflammatory Lung Disease by TNF $\alpha$ Gene Silencing in Macrophages. Mol. Pharm. 2014, 11, 4270-4279. [CrossRef] [PubMed]

21. Oglesby, I.K.; Chotirmall, S.H.; McElvaney, N.G.; Greene, C.M. Regulation of cystic fibrosis transmembrane conductance regulator by microRNA-145, -223 , and -494 is altered in $\triangle F 508$ cystic fibrosis airway epithelium. J. Immunol. 2013, 190, 3354-3362. [CrossRef] [PubMed]

22. Ramachandran, S.; Karp, P.H.; Osterhaus, S.R.; Jiang, P.; Wohlford-Lenane, C.; Lennox, K.A.; Jacobi, A.M.; Praekh, K.; Rose, S.D.; Behlke, M.A.; et al. Post-Transcriptional Regulation of Cystic Fibrosis Transmembrane Conductance Regulator Expression and Function by MicroRNAs. Am. J. Respir. Cell Mol. Biol. 2013, 49, 544-551. [CrossRef] [PubMed]

23. Amato, F.; Tomaiuolo, R.; Nici, F.; Borbone, N.; Elce, A.; Catalanotti, B.; D’Errico, S.; Morgillo, C.M.; de Rosa, G.; Mayol, L.; et al. Exploitation of a Very Small Peptide Nucleic Acid as a New Inhibitor of miR-509-3p Involved in the Regulation of Cystic Fibrosis Disease-Gene Expression. Biomed. Res. Int. 2014, 2014, 1-10. [CrossRef] [PubMed]

24. Viart, V.; Bergougnoux, A.; Bonini, J.; Varilh, J.; Chiron, R.; Tabary, O.; Molinari, N.; Claustres, M.; Taulan-Cadars, M. Transcription factors and miRNAs that regulate fetal to adult CFTR expression change are new targets for cystic fibrosis 2014. Eur. Respir. J. 2015, 45, 116-128. [CrossRef] [PubMed]

25. Staton, A.A.; Giraldez, A.J. Use of target protector morpholinos to analyze the physiological roles of specific miRNA-mRNA pairs in vivo. Nat. Protoc. 2011, 6, 2035-2049. [CrossRef] [PubMed]

26. Gruenweller, A.; Hartmann, R.K. Locked Nucleic Acid Oligonucleotides. Biol. Drugs 2007, 21, $235-243$. [CrossRef]

27. Rasmussen, S.; Roberts, P. Functional studies of microRNA based on knockdown using Locked Nucleic Acid probes. Nat. Methods 2007. [CrossRef]

28. Lopalco, A.; Ali, H.; Denora, N.; Rytting, E. Oxcarbazepine-loaded polymeric nanoparticles: Development and permeability studies across in vitro models of the blood-brain barrier and human placental trophoblast. Int. J. Nanomed. 2015, 10, 1985-1996. [CrossRef]

29. Fernández, F.E.; Bangel-Ruland, N.; Tomczak, K.; Weber, W.-M. Optimization of CFTR-mRNA transfection in human nasal epithelial cells. Transl. Med. Commun. 2016, 1, 5. [CrossRef]

30. Hardee, C.L.; Arévalo-Soliz, L.M.; Hornstein, B.D.; Zechiedrich, L. Advances in Non-Viral DNA Vectors for Gene Therapy. Genes 2017, 8, 65. [CrossRef] [PubMed]

31. Gaumet, M.; Vargas, A.; Gurny, R.; Delie, F. Nanoparticles for drug delivery: The need for precision in reporting particle size parameters. Eur. J. Pharm. Biopharm. 2008, 69, 1-9. [CrossRef] [PubMed]

32. Bose, S.; Tarafder, S. Calcium phosphate ceramic systems in growth factor and drug delivery for bone tissue engineering: A review. Acta Biomater. 2012. [CrossRef] [PubMed]

33. Kou, L.; Sun, J.; Zhai, Y.; He, Z. The endocytosis and intracellular fate of nanomedicines: Implication for rational design. Asian J. Pharm. Sci. 2013, 8, 1-8. [CrossRef]

34. Champion, J.A.; Walker, A.; Mitragotri, S. Role of Particle Size in Phagocytosis of Polymeric Microspheres. Pharm. Res. 2008, 25, 1815-1821. [CrossRef] [PubMed]

35. Malloy, A. Count, size and visualize nanoparticles. Mater. Today 2011, 14, 170-173. [CrossRef]

36. Filipe, V.; Hawe, A.; Jiskoot, W. Critical evaluation of Nanoparticle Tracking Analysis (NTA) by NanoSight for the measurement of nanoparticles and protein aggregates. Pharm. Res. 2010, 27, 796-810. [CrossRef] [PubMed]

37. Kapoor, M. Physicochemical characterization techniques for lipid based delivery systems for siRNA. Int. J. Pharm. 2012, 427, 35-57. [CrossRef] [PubMed] 
38. Liang, G.F.; Zhu, Y.L.; Sun, B.; Hu, F.H.; Tian, T.; Li, S.C.; Xiao, Z.D. PLGA-based gene delivering nanoparticle enhance suppression effect of miRNA in HePG2 cells. Nanoscale Res. Lett. 2011, 6, 447. [CrossRef] [PubMed]

39. Honary, S.; Zahir, F. Effect of zeta potential on the properties of nano-drug delivery systems-A review (Part 1). Trop. J. Pharm. Res. 2013, 12, 255-264. [CrossRef]

40. Colombo, S.; Cun, D.; Remaut, K.; Bunker, M.; Zhang, J.; Martin-Bertelsen, B.; Yaghmur, A.; Braeckmans, K.; Nielsen, H.M.; Foged, C. Mechanistic profiling of the siRNA delivery dynamics of lipid-polymer hybrid nanoparticles. J. Control. Release 2015, 201, 22-31. [CrossRef] [PubMed]

41. Moore, T.L.; Rodriguez-Lorenzo, L.; Hirsch, V.; Balog, S.; Urban, D.; Jud, C.; Rothen-Rutishauser, B.; Lattuadaa, M.; Petri-Fink, A. Nanoparticle colloidal stability in cell culture media and impact on cellular interactions. Chem. Soc. Rev. 2015, 44, 6287-6305. [CrossRef] [PubMed]

42. da Silva, A.L.; Cruz, F.F.; Rocco, P.R.M.; Morales, M.M. New perspectives in nanotherapeutics for chronic respiratory diseases. Biophys. Rev. 2017, 9, 793-803. [CrossRef] [PubMed]

43. Yhee, J.; Im, J.; Nho, R. Advanced Therapeutic Strategies for Chronic Lung Disease Using Nanoparticle-Based Drug Delivery. J. Clin. Med. 2016, 5, 82. [CrossRef] [PubMed]

44. Restani, R.B.; Silva, A.S.; Pires, R.F.; Cabral, R.; Correia, I.J.; Casimiro, T.; Bonifácio, V.D.B.; Aguiar-Ricardo, A. Nano-in-Micro POxylated Polyurea Dendrimers and Chitosan Dry Powder Formulations for Pulmonary Delivery. Part. Part. Syst. Charact. 2016, 33, 851-858. [CrossRef]

45. Silva, A.S.; Sousa, A.M.; Cabral, R.P.; Silva, M.C.; Costa, C.; Miguel, S.P.; Bonifácio, V.D.B.; Casimiro, T.; Correia, I.J.; Aguiar-Ricardo, A. Aerosolizable gold nano-in-micro dry powder formulations for theragnosis and lung delivery. Int. J. Pharm. 2017, 519, 240-249. [CrossRef] [PubMed]

46. Silva, M.; Silva, A.; Fernandez-Lodeiro, J.; Casimiro, T.; Lodeiro, C.; Aguiar-Ricardo, A. Supercritical $\mathrm{CO}_{2}$-Assisted Spray Drying of Strawberry-Like Gold-Coated Magnetite Nanocomposites in Chitosan Powders for Inhalation. Materials 2017, 10, 74. [CrossRef] [PubMed]

47. Manunta, M.D.I.; Tagalakis, A.D.; Attwood, M.; Aldossary, A.M.; Barnes, J.L.; Munye, M.M.; Weng, A.; McAnulty, R.J.; Hart, S.L. Delivery of ENaC siRNA to epithelial cells mediated by a targeted nanocomplex: A therapeutic strategy for cystic fibrosis. Sci. Rep. 2017, 7, 700. [CrossRef] [PubMed]

48. Alton, E.W.F.W.; Armstrong, D.K.; Ashby, D.; Bayfield, K.J.; Bilton, D.; Bloomfield, E.V.; Boyd, A.C.; Brand, J.; Buchan, R.; Calcedo, R.; et al. Repeated nebulisation of non-viral CFTR gene therapy in patients with cystic fibrosis: A randomised, double-blind, placebo-controlled, phase $2 \mathrm{~b}$ trial. Lancet Respir. Med. 2015. [CrossRef]

49. Dugernier, J.; Reychler, G.; Wittebole, X.; Roeseler, J.; Depoortere, V.; Sottiaux, T.; Michotte, J.-B.; Vanbever, R.; Dugernier, T.; Goffette, P.; et al. Aerosol delivery with two ventilation modes during mechanical ventilation: A randomized study. Ann. Intensive Care 2016, 6, 73. [CrossRef] [PubMed]

50. Dugernier, J.; Hesse, M.; Vanbever, R.; Depoortere, V.; Roeseler, J.; Michotte, J.-B.; Laterre, P.-F.; Jamar, F.; Reychler, G. SPECT-CT Comparison of Lung Deposition using a System combining a Vibrating-mesh Nebulizer with a Valved Holding Chamber and a Conventional Jet Nebulizer: A Randomized Cross-over Study. Pharm. Res. 2017, 34, 290-300. [CrossRef] [PubMed]

51. Wacker, M. Nanocarriers for intravenous injection-The long hard road to the market. Int. J. Pharm. 2013, 457, 50-62. [CrossRef] [PubMed]

52. Hines, D.J.; Kaplan, D.L. Poly(lactic-co-glycolic) acid-controlled-release systems: Experimental and modeling insights. Crit. Rev. Ther. Drug Carr. Syst. 2013, 30, 257-276. [CrossRef] [PubMed]

53. Günday Türeli, N.; Torge, A.; Juntke, J.; Schwarz, B.C.; Schneider-Daum, N.; Türeli, A.E.; Lehr, C.M.; Schneider, M. Ciprofloxacin-loaded PLGA nanoparticles against cystic fibrosis P. aeruginosa lung infections. Eur. J. Pharm. Biopharm. 2017, 117, 363-371. [CrossRef] [PubMed]

54. Akbuga, J.; Ozbas-Turan, S.; Ekentok, C. Chitosan Nanoparticles in Gene Delivery. In Percutaneous Penetration Enhancers Chemical Methods in Penetration Enhancement; Springer: Berlin/Heidelberg, Germany, 2016; pp. 337-351.

55. Cun, D.; Jensen, D.K.; Maltesen, M.J.; Bunker, M.; Whiteside, P.; Scurr, D.; Foged, C.; Nielsen, H.M. High loading efficiency and sustained release of siRNA encapsulated in PLGA nanoparticles: Quality by design optimization and characterization. Eur. J. Pharm. Biopharm. 2011, 77, 26-35. [CrossRef] [PubMed] 
56. Menchicchi, B.; Fuenzalida, J.P.; Hensel, A.; Swamy, M.J.; David, L.; Rochas, C.; Goycoolea, F.M. Biophysical analysis of the molecular interactions between polysaccharides and mucin. Biomacromolecules 2015, 16, 924-935. [CrossRef] [PubMed]

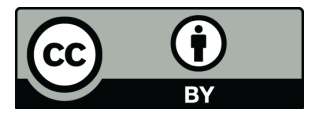

(C) 2018 by the authors. Licensee MDPI, Basel, Switzerland. This article is an open access article distributed under the terms and conditions of the Creative Commons Attribution (CC BY) license (http://creativecommons.org/licenses/by/4.0/). 